\title{
A Fast Implicit Finite Difference Method for Fractional Advection-Dispersion Equations with Fractional Derivative Boundary Conditions
}

\author{
Taohua Liu and Muzhou Hou \\ School of Mathematics and Statistics, Central South University, Changsha, Hunan 410083, China \\ Correspondence should be addressed to Muzhou Hou; houmuzhou@sina.com
}

Received 4 June 2017; Revised 16 August 2017; Accepted 22 August 2017; Published 24 September 2017

Academic Editor: Mariano Torrisi

Copyright (C) 2017 Taohua Liu and Muzhou Hou. This is an open access article distributed under the Creative Commons Attribution License, which permits unrestricted use, distribution, and reproduction in any medium, provided the original work is properly cited.

Fractional advection-dispersion equations, as generalizations of classical integer-order advection-dispersion equations, are used to model the transport of passive tracers carried by fluid flow in a porous medium. In this paper, we develop an implicit finite difference method for fractional advection-dispersion equations with fractional derivative boundary conditions. First-order consistency, solvability, unconditional stability, and first-order convergence of the method are proven. Then, we present a fast iterative method for the implicit finite difference scheme, which only requires storage of $O(K)$ and computational cost of $O(K \log K)$. Traditionally, the Gaussian elimination method requires storage of $O\left(K^{2}\right)$ and computational cost of $O\left(K^{3}\right)$. Finally, the accuracy and efficiency of the method are checked with a numerical example.

\section{Introduction}

Fractional derivatives are almost as old as their more familiar integer-order counterparts [1,2]. Fractional advectiondispersion equations are used to model many problems in physics, biology, and finance [3-5]. Fractional differential equations have attracted fantastic attention of many authors in recent years [6-11].

The fractional space derivatives are used to establish anomalous diffusion and dispersion models, where the propagation velocity of the particle beam is different from the classical Brown motion model. When the space second derivative in a diffusion or dispersion model is replaced with a fractional space derivative, it leads to superdiffusion. In this paper, we consider a one-dimensional fractional advection-dispersion equation with fractional derivative boundary conditions:

$$
\begin{array}{r}
\frac{\partial p(r, t)}{\partial t}=-v(r) \frac{\partial p(r, t)}{\partial r}+d(r) \frac{\partial^{\alpha} p(r, t)}{\partial r^{\alpha}}+f(r, t), \\
0<r<R, 0<t,
\end{array}
$$

$$
\begin{array}{ll}
p(0, t)=0, & \\
\beta p(R, t)+\left.\left(d(r) \frac{\partial^{\alpha-1} p(r, t)}{\partial r^{\alpha-1}}\right)\right|_{r=R}=w(t), & 0<t, \\
p(r, 0)=u(r), \quad 0 \leq r \leq R, &
\end{array}
$$

where $1<\alpha \leq 2 ; p(r, t)$ is the distribution function of the diffusion quantity; $v(r)$ is flow velocity; $d(r)$ is diffusion coefficient; $f(r, t)$ is the source term. We assume $v(r) \geq 0$ and $d(r) \geq 0$, so that the flow is from left to right. The space fractional partial derivatives in (1) and (2) are left-sided Riemann-Liouville fractional derivatives over the $x$ domain. For a function $g(x)$ over the interval $L<x<R$, the left-sided Riemann-Liouville fractional derivative of order $\mu$ is defined by

$$
\frac{d^{\mu} g(x)}{d x^{\mu}}=\frac{1}{\Gamma(n-\mu)} \frac{d^{n}}{d x^{n}} \int_{L}^{x} \frac{g(\xi)}{(x-\xi)^{\mu+1-n}} d \xi
$$


where $n$ is an integer, such that $n-1<\mu \leq n$. See [12] for details. If $\mu$ is an integer, then (4) gives the standard integer derivative. $\beta=0$ corresponds to a fractional Neumann boundary condition and $\beta>0$ corresponds to a fractional Robin boundary condition. In this paper, we only discuss $\beta>0$.

For a one-dimensional advection-dispersion equation with constant coefficients, Benson et al. [13] got available analytical solutions using Fourier transform method. However, many problems require a model with variable coefficients. Meerschaert and Tadjeran [14] developed finite difference approximations for one-dimensional fractional advection-dispersion equations with Dirichlet boundary conditions. And, recently, some authors have discussed numerical method for fractional equations with fractional derivative boundary conditions. Jia and Wang [15] developed fast implicit finite difference methods for two-sided space fractional diffusion equations with fractional derivative boundary conditions. Guo et al. [16] developed implicit finite difference method for fractional percolation equation with Dirichlet and fractional boundary conditions. As far as we know, the study on numerical method for one-dimensional advection-dispersion equations with fractional derivative boundary conditions is still limited. This motivates us to examine a fast implicit finite difference method for fractional advection-dispersion equations with fractional derivative boundary conditions in this paper.

The rest of the paper is organized as follows. In Section 2, we develop an implicit finite difference method and analyze its consistency. In Section 3, the solvability, unconditional stability, and convergence of the method are proven. In Section 4 , we present a fast iterative method for the implicit finite difference scheme, which only requires storage of $O(K)$ and computational cost of $O(K \log K)$. In Section 5, a numerical experiment is presented to verify the accuracy and efficiency of the proposed scheme.

\section{The Implicit Finite Difference Method for the Advection-Dispersion Equations with Fractional Derivative Boundary Conditions and Its Consistency}

First, let $h=R / K$ and $\Delta t$ be the spatial mesh width and the time step, where $K$ is a positive integer. We define a spatial partition by $r_{i}=i h$ for $i=0,1, \ldots, K$ and a temporal partition by $t_{n}=n \Delta t$ for $n=0,1, \ldots, N$. Let $v_{i}=v\left(r_{i}\right), d_{i}=d\left(r_{i}\right)$, $f_{i}^{n}=f\left(r_{i}, t_{n}\right), w^{n}=w\left(t_{n}\right), u_{i}=u\left(r_{i}\right)$. Denote the exact and numerical solutions at the mesh point $\left(r_{i}, t_{n}\right)$ by $P_{i}^{n}=p\left(r_{i}, t_{n}\right)$ and $p_{i}^{n}$, respectively. $P_{i}^{0}=u_{0}\left(r_{i}\right)$ and $p_{0}^{n}=0$.

Then, we employ the backward Euler scheme to approximate the first-order time derivative; a right-shifted Grünwald formula to approximate left-sided $\alpha$-order Riemann-Liouville fractional derivative and standard Grünwald formula to approximate $\alpha$ - 1-order RiemannLiouville fractional derivative are defined as follows [12]:

$$
\begin{aligned}
& \left.\frac{\partial p}{\partial t}\right|_{\left(r_{i}, t_{n+1}\right)}=\frac{P_{i}^{n+1}-P_{i}^{n}}{\Delta t}+O(\Delta t), \\
& \left.\frac{\partial p}{\partial r}\right|_{\left(r_{i}, t_{n+1}\right)}=\frac{P_{i}^{n+1}-P_{i-1}^{n+1}}{h}+O(h),
\end{aligned}
$$

$$
\begin{aligned}
\left.\frac{\partial^{\alpha} p}{\partial r^{\alpha}}\right|_{\left(r_{i}, t_{n+1}\right)} & =\frac{1}{h^{\alpha}} \sum_{j=0}^{i+1} g_{\alpha, j} P_{i-j+1}^{n+1}+O(h), \\
\left.\frac{\partial^{\alpha-1} p}{\partial r^{\alpha-1}}\right|_{\left(R, t_{n+1}\right)} & =\frac{1}{h^{\alpha-1}} \sum_{j=0}^{K} g_{\alpha-1, j} P_{K-j}^{n+1}+O(h),
\end{aligned}
$$

where $g_{\delta, l},(\delta=\alpha-1 ; \alpha)$ is normalized Grünwald weights:

$$
g_{\delta, l}=\frac{\Gamma(l-\delta)}{\Gamma(-\delta) \Gamma(l+1)} .
$$

Moreover, the normalized Grünwald weights satisfy the properties in the following Lemma 1.

Lemma 1. Let $\mu$ be a positive real number and the integer $n \geq$ 1. We have

(i) $g_{\mu, 0}=1, g_{\mu, j}=(1-(\mu+1) / j) g_{\mu, j-1}$ for $j \geq 1$;

(ii) $g_{\mu, 1}<g_{\mu, 2}<\cdots<0, \sum_{j=0}^{n} g_{\mu, j}>0$ for $0<\mu<1$;

(iii) $g_{\mu, 2}>g_{\mu, 3}>\cdots>0, \sum_{j=0}^{n} g_{\mu, j}<0$ for $1<\mu<2$.

Therefore implicit finite difference method for the advection-dispersion equations with fractional derivative boundary conditions (1)-(3) can be written as follows:

$$
\begin{aligned}
& \frac{p_{i}^{n+1}-p_{i}^{n}}{\Delta t} \\
& =\left(-v_{i} \frac{p_{i}^{n+1}-p_{i-1}^{n+1}}{h}+\frac{d_{i}}{h^{\alpha}} \sum_{j=0}^{i+1} g_{\alpha, k} p_{i-j+1}^{n+1}\right)+f_{i}^{n}, \\
& \quad \text { for } i=1,2, \ldots, K-1,
\end{aligned}
$$

$p_{0}^{n}=0$,

$\beta p_{K}^{n+1}+\left(\frac{d_{K}}{h^{\alpha-1}} \sum_{j=0}^{K} g_{\alpha-1, j} p_{K-j}^{n+1}\right)=w^{n+1}$,

$p_{i}^{0}=u_{i}$

Denote the local truncation error by $R_{i}^{n+1}$ for $1 \leq i \leq K$. Then, from (7), we have

$$
\begin{aligned}
R_{i}^{n+1}= & \frac{P_{i}^{n+1}-P_{i}^{n}}{\Delta t} \\
& -\left(-v_{i} \frac{P_{i}^{n+1}-P_{i-1}^{n+1}}{h}+\frac{d_{i}}{h^{\alpha}} \sum_{k=0}^{i+1} g_{\alpha, k} P_{i-k+1}^{n+1}\right)-f_{i}^{n} \\
= & O(\Delta t)+O(h), \quad 1 \leq i \leq K-1, \\
R_{N}^{n+1}= & \beta P_{K}^{n+1}-\left(\frac{d_{K}}{h^{\alpha-1}} \sum_{j=0}^{K} g_{\alpha-1, j} P_{K-j}^{n+1}\right)-w^{n+1} \\
= & O(h) .
\end{aligned}
$$


From (8), this implicit finite difference scheme is consistent. Equations (7) may be rearranged as follows:

$$
\begin{aligned}
& -g_{\alpha, 0} B_{i} p_{i+1}^{n+1}+\left(1+E_{i}-g_{\alpha, 1} B_{i}\right) p_{i}^{n+1} \\
& -\left(E_{i}+g_{\alpha, 2} B_{i}\right) p_{i-1}^{n+1}-B_{i} \sum_{k=3}^{i+1} g_{\alpha, k} p_{i-k+1}^{n+1}=p_{i}^{n} \\
& \quad+\Delta t f_{i}^{n+1}, \\
& \left(\beta h^{\alpha-1}+d_{K} g_{\alpha-1,0}\right) p_{K}^{n+1} \\
& \quad+\left(d_{K} \sum_{j=1}^{K-1} g_{\alpha-1, j} p_{K-j}^{n+1}\right)=h^{\alpha-1} w^{n+1},
\end{aligned}
$$

where $B_{i}=d_{i} \Delta t / h^{\alpha}, E_{i}=v_{i} \Delta t / h$. Equations (9) can be written in matrix form:

$$
\mathbf{A} \mathbf{p}^{n+1}=\mathbf{q}^{n}+\mathbf{f}^{n+1}
$$

where

$$
\begin{aligned}
\mathbf{p}^{n+1} & =\left(p_{1}^{n+1}, p_{2}^{n+1}, \ldots, p_{K}^{n+1}\right)^{T}, \\
\mathbf{q}^{n} & =\left(p_{1}^{n}, p_{2}^{n}, \ldots, p_{K-1}^{n}, 0\right)^{T}, \\
\mathbf{f}^{n+1} & =\left(\Delta t f_{1}^{n+1}, \Delta t f_{2}^{n+2}, \ldots, \Delta t f_{K-1}^{n+1}, h^{\alpha-1} w^{n+1}\right)^{T},
\end{aligned}
$$

where the coefficient matrix $\mathbf{A}=\left[a_{i, j}\right]_{K \times K}$ and its entries are

$$
\begin{aligned}
& a_{i, j} \\
& \quad \begin{cases}-g_{\alpha, i+1-j} B_{i}, & \text { for } 1 \leq j \leq i-2,1 \leq i \leq K-1 \\
-E_{i}-g_{\alpha, 2} B_{i}, & \text { for } 1 \leq j=i-1 \leq K-1 ; \\
1+E_{i}-g_{\alpha, 1} B_{i}, & \text { for } j=i, 1 \leq i \leq K-1 ; \\
-g_{\alpha, 0} B_{i}, & \text { for } j=i+1,1 \leq i \leq K-1 ; \\
0, & \text { for } j=i+2,1 \leq i \leq K-1 \\
g_{\alpha-1, K-j} d_{K}, & \text { for } 1 \leq j \leq K-1, i=K \\
\beta h^{\alpha-1}+d_{K} g_{\alpha-1,0}, & \text { for } i=j=K .\end{cases}
\end{aligned}
$$

\section{Solvability, Stability, and Convergence Analysis}

Having developed a numerical scheme and shown that it consistent, in the following theorems, we show this method is solvable, unconditionally stable, and convergent.

Theorem 2 (solvability). If $\beta>0,(10)$ is uniquely solvable.
Proof. Let $r_{i}$ be the sum of absolute values of elements along the $i$ th row excluding the diagonal elements $A_{i, i}$. According to Lemma 1, we can get

$$
\begin{aligned}
r_{i} & =\sum_{j=1, j \neq i}^{K}\left|A_{i, j}\right| \\
& =\sum_{j=1}^{i-2}\left|-B_{i} g_{i+1-j}^{(\alpha)}\right|+\left|E_{i}+B_{i} g_{2}^{(\alpha)}\right|+\left|B_{i} g_{0}^{(\alpha)}\right| \\
& =\left(\sum_{j=0}^{i} g_{j}^{(\alpha)}-g_{1}^{(\alpha)}\right) B_{i}+E_{i}<-g_{1}^{(\alpha)} B_{i}<A_{i, i} \\
r_{K} & =\sum_{j=1}^{K-1}\left|A_{K, j}\right|=\sum_{j=1}^{K-1}\left|d_{K} g_{K-j}^{(\alpha-1)}\right|=-\sum_{j=1}^{K-1} d_{K} g_{j}^{(\alpha-1)} \\
& =-d_{K}\left(\sum_{j=0}^{K-1} g_{j}^{(\alpha-1)}-g_{0}^{(\alpha-1)}\right)<d_{K} g_{0}^{(\alpha-1)}<A_{K, K} .
\end{aligned}
$$

Matrix $A$ is strictly diagonal, which guarantees the invertibility of matrix $A$. Hence, (10) is uniquely solvable.

To discuss the stability of the numerical method, we denote by $\widetilde{p}_{i}^{n}(1 \leq i \leq K)$ the approximate solution of the difference scheme with the initial condition $\widetilde{p}_{i}^{0}(1 \leq i \leq K)$ and define $\varepsilon_{i}^{n}=p_{i}^{n}-\widetilde{p}_{i}^{n}, \varepsilon^{n}=\left(\varepsilon_{1}^{n}, \varepsilon_{2}^{n}, \ldots, \varepsilon_{K}^{n}\right)^{T},\left\|\varepsilon^{n}\right\|_{\infty}=$ $\max _{1 \leq i \leq K}\left|\varepsilon_{i}^{n}\right|$.

Theorem 3. The implicit finite difference method for the advection-dispersion equations with fractional derivative boundary conditions defined by (7) is unconditionally stable.

Proof. From (9) and the definition of $\varepsilon_{i}^{n}$, we have

$$
\begin{aligned}
& -g_{\alpha, 0} B_{i} \varepsilon_{i+1}^{n+1}+\left(1+E_{i}-g_{\alpha, 1} B_{i}\right) \varepsilon_{i}^{n+1} \\
& -\left(E_{i}+g_{\alpha, 2} B_{i}\right) \varepsilon_{i-1}^{n+1}-B_{i} \sum_{k=3}^{i+1} g_{\alpha, k} \varepsilon_{i-k+1}^{n+1}=\varepsilon_{i}^{n}, \\
& \left(\beta h^{\alpha-1}+d_{K} g_{\alpha-1,0}\right) \varepsilon_{K}^{n+1}+\left(d_{K} \sum_{j=1}^{K-1} g_{\alpha-1, j} \varepsilon_{K-j}^{n+1}\right)=0 .
\end{aligned}
$$

By Lemma 1, we have

$$
\sum_{j=1}^{K-1}\left|g_{\alpha-1, j}\right|=-\sum_{j=1}^{K-1} g_{\alpha-1, j}<g_{\alpha-1,0} .
$$

It follows from (15) that

$$
\begin{aligned}
\left|\varepsilon_{K}^{n+1}\right| & =\frac{\left|d_{K} \sum_{j=1}^{K-1} g_{\alpha-1, j} \varepsilon_{K-j}^{n+1}\right|}{\left|h^{\alpha-1} \beta+g_{\alpha-1,0} d_{K}\right|} \\
& \leq \frac{d_{K} \sum_{j=1}^{K-1}\left|g_{\alpha-1, j}\right|\left|\varepsilon_{K-j}^{n+1}\right|}{h^{\alpha-1} \beta+g_{\alpha-1,0} d_{K}}<\max _{1 \leq i \leq K-1}\left|\varepsilon_{i}^{n}\right| .
\end{aligned}
$$


Therefore, we suppose that $\left\|\varepsilon^{n}\right\|_{\infty}=\left|\varepsilon_{i_{0}}^{n}\right|$, where $1 \leq i_{0} \leq$ $N-1$. By Lemmas 1(i) and (iii), then

$$
\begin{aligned}
& \left\|\varepsilon^{n+1}\right\|_{\infty}=\left|\varepsilon_{i_{0}}^{n+1}\right|<\left(1+E_{i_{0}}-E_{i_{0}}-\sum_{k=0}^{i_{0}+1} g_{\alpha, k} B_{i_{0}}\right)\left|\varepsilon_{i_{0}}^{n+1}\right| \\
& =\left(1+E_{i_{0}}-g_{\alpha, 1} B_{i_{0}}\right)\left|\varepsilon_{i_{0}}^{n+1}\right|-\left(g_{\alpha, 0} B_{i_{0}}\right)\left|\varepsilon_{i_{0}}^{n+1}\right|-\left(E_{i_{0}}\right. \\
& \left.+g_{\alpha, 2} B_{i_{0}}\right)\left|\varepsilon_{i_{0}}^{n+1}\right|-\left(\sum_{k=3}^{i_{0}+1} g_{\alpha, k} B_{i_{0}}\right)\left|\varepsilon_{i_{0}}^{n+1}\right| \leq\left(1+E_{i_{0}}\right. \\
& \left.-g_{\alpha, 1} B_{i_{0}}\right)\left|\varepsilon_{i_{0}}^{n+1}\right|-\left(g_{\alpha, 0} B_{i_{0}}\right)\left|\varepsilon_{i_{0}+1}^{n+1}\right|-\left(E_{i_{0}}+g_{\alpha, 2} B_{i_{0}}\right) \\
& \cdot\left|\varepsilon_{i_{0}-1}^{n+1}\right|-\left(\sum_{k=3}^{i_{0}+1} g_{\alpha, k} B_{i_{0}}\right)\left|\varepsilon_{i_{0}-k+1}^{n+1}\right| \\
& \leq \mid\left(1+E_{i_{0}}-g_{\alpha, 1} B_{i_{0}}\right) \varepsilon_{i_{0}}^{n+1}-\left(g_{\alpha, 0} B_{i_{0}}\right) \varepsilon_{i_{0}+1}^{n+1} \\
& -\left(E_{i_{0}}+g_{\alpha, 2} B_{i_{0}}\right) \varepsilon_{i_{0}-1}^{n+1}-\left(\sum_{k=3}^{i_{0}+1} g_{\alpha, k} B_{i_{0}}\right) \varepsilon_{i_{0}-k+1}^{n+1} \mid \\
& =\left|\varepsilon_{i_{0}}^{n}\right| \leq\left\|\varepsilon^{n}\right\|_{\infty},
\end{aligned}
$$

which implies that $\left\|\varepsilon^{n}\right\|_{\infty}<\left\|\varepsilon^{0}\right\|_{\infty}$.

Thus, the implicit finite difference method defined by (7) is unconditionally stable.

For the convergence analysis, we define $e_{i}^{n}=P_{i}^{n}-p_{i}^{n}(1 \leq$ $i \leq K)$ and $\mathbf{e}^{n}=\left(e_{1}^{n}, e_{2}^{n}, \ldots, e_{K}^{n}\right)^{T},\left\|\mathbf{e}^{n}\right\|_{\infty}=\max _{1 \leq i \leq K}\left|\mathbf{e}_{i}^{n}\right|$.

Theorem 4. There exists a positive constant $C$, independent of $h$ and $\Delta t$, such that

$$
\left\|\mathbf{e}^{n+1}\right\|_{\infty} \leq C(\Delta t+h), \quad 1 \leq n \leq N .
$$

Proof. From (9), we have

$$
\begin{aligned}
& -g_{\alpha, 0} B_{i} e_{i+1}^{n+1}+\left(1+E_{i}-g_{\alpha, 1} B_{i}\right) e_{i}^{n+1} \\
& -\left(E_{i}+g_{\alpha, 2} B_{i}\right) e_{i-1}^{n+1}-B_{i} \sum_{k=3}^{i+1} g_{\alpha, k} e_{i-k+1}^{n+1}=e_{i}^{n} \\
& +\Delta t R_{i}^{n+1}, \\
& \left(\beta h^{\alpha-1}+d_{K} g_{\alpha-1,0}\right) e_{K}^{n+1} \\
& \quad+\left(d_{K} \sum_{j=0}^{K-1} g_{\alpha-1, j} e_{K-j}^{n+1}\right)=h^{\alpha-1} R_{K}^{n+1},
\end{aligned}
$$

with $e_{0}^{n+1}=0$, and $e_{i}^{0}=0, i=0,1, \ldots, K$. If $\left\|\mathbf{e}^{n+1}\right\|_{\infty}=\left|e_{K}^{n+1}\right| \geq$ $\max _{1 \leq i \leq K-1}\left|e_{i}^{n+1}\right|$, we have

$$
\begin{aligned}
& \left(\beta h^{\alpha-1}+d_{K} g_{\alpha-1,0}\right) e_{K}^{n+1}+\left(d_{K} \sum_{j=0}^{K-1} g_{\alpha-1, j} e_{K-j}^{n+1}\right) \\
& =h^{\alpha-1} R_{K}^{n+1} .
\end{aligned}
$$

By Lemma 1(ii), then

$$
\begin{aligned}
& \beta h^{\alpha-1}+d_{K} \sum_{j=0}^{K-1} g_{\alpha-1, j}\left|e_{K}^{n+1}\right| \\
& =\left(\beta h^{\alpha-1}+d_{K} g_{\alpha-1,0}\right)\left|e_{K}^{n+1}\right|-d_{K} \sum_{j=1}^{K-1}\left|g_{\alpha-1, j}\right|\left|e_{K}^{n+1}\right| \\
& \leq\left(\beta h^{\alpha-1}+d_{K} g_{\alpha-1,0}\right)\left|e_{K}^{n+1}\right|-d_{K} \sum_{j=1}^{K-1}\left|g_{\alpha-1, j}\right|\left|e_{K-j}^{n+1}\right| \\
& \leq\left(\beta h^{\alpha-1}+d_{K} g_{\alpha-1,0}\right)\left|e_{K}^{n+1}\right|-d_{K} \sum_{j=1}^{K-1}\left|g_{\alpha-1, j} e_{K-j}^{n+1}\right| \\
& \leq\left|\left(\beta h^{\alpha-1}+d_{K} g_{\alpha-1,0}\right) e_{K}^{n+1}+d_{K} \sum_{j=1}^{K-1} g_{\alpha-1, j} e_{K-j}^{n+1}\right| \\
& =h^{\alpha-1}\left|R_{K}^{n+1}\right| .
\end{aligned}
$$

With Lemma 1 and the Stirling formula for the Gamma function [17], we have

$$
\begin{aligned}
\left(\sum_{j=0}^{K-1} g_{\alpha-1, j}\right)^{-1} & =(-1)^{K-1}\left(\begin{array}{c}
\alpha-2 \\
K-1
\end{array}\right)^{-1} \\
& =\frac{\Gamma(K)}{\Gamma(2-\alpha) \Gamma(K-\alpha+1)} \\
& =O\left(K^{\alpha-1}\right),
\end{aligned}
$$

as $K \rightarrow \infty$. Combining (22) and (23), we get

$$
\left|e_{K}^{n+1}\right| \leq\left(d_{K} \sum_{j=0}^{K-1} g_{\alpha-1, j}\right)^{-1} h^{\alpha-1}\left|R_{K}^{n+1}\right| \leq C_{1} h
$$

If $\left\|\mathbf{e}^{n+1}\right\|_{\infty}=\left|e_{i_{0}}^{n+1}\right|>\left|e_{K}^{n+1}\right|, 1 \leq i_{0} \leq K-1$, then

$$
\begin{array}{r}
\left\|\mathbf{e}^{n+1}\right\|_{\infty}=\left|e_{i_{0}}^{n+1}\right|<\left(1+E_{i_{0}}-E_{i_{0}}-\sum_{k=0}^{i_{0}+1} g_{\alpha, k} B_{i_{0}}\right) \\
\cdot\left|e_{i_{0}}^{n+1}\right|=\left(1+E_{i_{0}}-g_{\alpha, 1} B_{i_{0}}\right)\left|e_{i_{0}}^{n+1}\right|-\left(g_{\alpha, 0} B_{i_{0}}\right) \\
\cdot\left|e_{i_{0}}^{n+1}\right|-\left(E_{i_{0}}+g_{\alpha, 2} B_{i_{0}}\right)\left|e_{i_{0}}^{n+1}\right|-\left(\sum_{k=3}^{i_{0}+1} g_{\alpha, k} B_{i_{0}}\right) \\
\cdot\left|e_{i_{0}}^{n+1}\right| \leq\left(1+E_{i_{0}}-g_{\alpha, 1} B_{i_{0}}\right)\left|e_{i_{0}}^{n+1}\right|-\left(g_{\alpha, 0} B_{i_{0}}\right) \\
\cdot\left|e_{i_{0}+1}^{n+1}\right|-\left(E_{i_{0}}+g_{\alpha, 2} B_{i_{0}}\right)\left|e_{i_{0}-1}^{n+1}\right|-\left(\sum_{k=3}^{i_{0}+1} g_{\alpha, k} B_{i_{0}}\right) \\
\cdot\left|e_{i_{0}-k+1}^{n+1}\right| \leq \mid\left(1+E_{i_{0}}-g_{\alpha, 1} B_{i_{0}}\right) e_{i_{0}}^{n+1}
\end{array}
$$




$$
\begin{aligned}
& -\left(g_{\alpha, 0} B_{i_{0}}\right) e_{i_{0}+1}^{n+1}-\left(E_{i_{0}}+g_{\alpha, 2} B_{i_{0}}\right) e_{i_{0}-1}^{n+1} \\
& -\left(\sum_{k=3}^{i_{0}+1} g_{\alpha, k} B_{i_{0}}\right) e_{i_{0}-k+1}^{n+1}|=| e_{i_{0}}^{n}+\Delta t R_{i_{0}}^{n+1} \mid \\
& \leq \max _{1 \leq i \leq K-1}\left|e_{i}^{n}\right|+\Delta t C_{2}(\Delta t+h) .
\end{aligned}
$$

By induction, we can finally obtain

$$
\left\|\mathbf{e}^{n}\right\|_{\infty} \leq C_{1} h+n \Delta t C_{2}(\Delta t+h) \leq C(\Delta t+h) .
$$

This completes the proof.

\section{Storage and Fast Krylov Subspace Method of the Coefficient Matrix}

The following theorem shows that the storage of matrix $A$ can be stored in $O(K)$ memories, instead of $O\left(K^{2}\right)$ memories.

Theorem 5. Coefficient matrix $A$ can be stored in $4 K+1$ memories.

Proof. We express coefficient matrix $A$ defined by (12) in block form as follows:

$$
A=\left[\begin{array}{cc}
A_{K-1, K-1} & A_{K-1, K} \\
A_{K, K-1}^{T} & a_{K, K}
\end{array}\right],
$$

where $A_{K-1, K-1}$ is the $(K-1) \times(K-1)$ matrix that consists of the first $K-1$ rows and the first $K-1$ columns. $A_{K, K-1}^{T}$ is an $(K-1)$-dimensional row vector that consists of the first $K-1$ entries in the $K$ th row of matrix $A$, and $A_{K-1, K}$ is an $(K-1)$-dimensional column vector that consists of the first $K-1$ entries in the $K$ th column of matrix $A$. Matrix $A_{K-1, K-1}$ has the similar form as that in the finite difference method for (1)-(3) with the fractional derivative boundary conditions [15]. $A_{K-1, K-1}$ can be shown to have the following decomposition:

$$
\begin{aligned}
A_{K-1, K-1}= & I-\operatorname{diag}\left(\left\{B_{i}\right\}_{i=1}^{K-1}\right) \cdot A^{(\alpha)} \\
& +\operatorname{diag}\left(\left\{E_{i}\right\}_{i=1}^{K-1}\right) \cdot A^{(\beta)},
\end{aligned}
$$

where $I$ is the identity matrix of the order $K-1$. And $A^{(\alpha)}$ and $A^{(\beta)}$ are $(N-1)$ th-order Toeplitz matrix given as follows:

$$
A^{(\alpha)}=\left[\begin{array}{cccccc}
g_{\alpha, 1} & g_{\alpha, 0} & 0 & \cdots & 0 & 0 \\
g_{\alpha, 2} & g_{\alpha, 1} & g_{\alpha, 0} & \ddots & 0 & 0 \\
g_{\alpha, 3} & g_{\alpha, 2} & g_{\alpha, 1} & \ddots & 0 & 0 \\
\vdots & \ddots & \ddots & \ddots & \ddots & \vdots \\
g_{\alpha, K-2} & g_{\alpha, K-3} & g_{\alpha, K-4} & \ddots & g_{\alpha, 1} & g_{\alpha, 0} \\
g_{\alpha, K-1} & g_{\alpha, K-2} & g_{\alpha, K-3} & \cdots & g_{\alpha, 2} & g_{\alpha, 1}
\end{array}\right],
$$

$$
A^{(\beta)}=\left[\begin{array}{cccccc}
1 & 0 & 0 & \cdots & 0 & 0 \\
-1 & 1 & 0 & \ddots & 0 & 0 \\
0 & -1 & 1 & \ddots & 0 & 0 \\
\vdots & \ddots & \ddots & \ddots & \ddots & \vdots \\
0 & 0 & 0 & \ddots & 1 & 0 \\
0 & 0 & 0 & \cdots & -1 & 1
\end{array}\right] .
$$

According to the definition of (12), we can write $A_{K-1, K}$ and $A_{K, K-1}^{T}$ as follows:

$$
\begin{aligned}
& A_{K-1, K}=-B_{K-1} \cdot g_{\alpha, 0} \cdot I_{K-1}, \\
& A_{K, K-1}^{T}=d_{K} \cdot g^{(\alpha)}
\end{aligned}
$$

where $g^{(\alpha)}=\left(g_{\alpha, K-1}, g_{\alpha, K-2}, \ldots, g_{\alpha, 1}\right)$, and $I_{K-1}$ is the $(K-$ 1) th column of $I$.

Thus, in order to store $A$, we need only to store $\left(B_{1}, B_{2}, \ldots, B_{K-1}\right)^{T},\left(E_{1}, E_{2}, \ldots, E_{K-1}\right)^{T}, \quad\left(g_{\alpha, 0}, g_{\alpha, 1}, \ldots\right.$, $\left.g_{\alpha, K-1}\right)^{T}, g^{(\beta)}=(1,-1, \ldots, 0)^{T}, A_{K, K-1}=\left(a_{K, 1}, a_{K, 2}, \ldots\right.$, $\left.a_{K, K-1}\right)^{T}, I$, and $a_{K, K}$, which totally have $4 K+1$ parameters, instead of storing the super triangular matrix $A$ in a straightforward but naive way that required $K^{2}$ memories.

Theorem 6. For any vector $\mu_{K} \in R^{K}$ and $T_{K}$ to be a Toeplitz matrix of order $K$, the matrix-vector multiplication $T_{K} \mu_{K}$ can be carried out in $O(K \log K)$ operations without any lossy compression.

Proof. A Toeplitz matrix is a matrix in which each descending diagonal from left to right is constant. Let $T_{K}$ be a Toeplitz matrix of order $K$ of the following form:

$$
T_{K}=\left[\begin{array}{cccccc}
t_{0} & t_{-1} & t_{-2} & \cdots & t_{2-K} & t_{1-K} \\
t_{1} & t_{0} & t_{-1} & \ddots & t_{3-K} & t_{2-K} \\
t_{2} & t_{1} & t_{0} & \ddots & \ddots & t_{3-K} \\
\vdots & \ddots & \ddots & \ddots & \ddots & \vdots \\
t_{K-2} & t_{K-3} & \ddots & \ddots & t_{0} & t_{-1} \\
t_{K-1} & t_{K-2} & t_{K-3} & \cdots & t_{1} & t_{0}
\end{array}\right] .
$$

The matrix-vector multiplication $T_{K} \mu_{K}$ can be carried out via the fast Fourier transform (FFT). First, we embed Toeplitz matrix $T_{K}$ into a $2 K \times 2 K$ circulant matrix $C_{2 N}$ as follows $[18,19]$ :

$$
C_{2 K}=\left[\begin{array}{ll}
T_{K} & B_{K} \\
B_{K} & T_{K}
\end{array}\right],
$$


TABLE 1: Error behavior for the implicit finite difference scheme (7), for example, at time $T=1$ and $\beta=1.0$.

\begin{tabular}{|c|c|c|c|c|c|c|}
\hline \multirow{2}{*}{$\Delta t=h$} & \multicolumn{2}{|c|}{$\alpha=1.9$} & \multicolumn{2}{|c|}{$\alpha=1.8$} & \multicolumn{2}{|c|}{$\alpha=1.6$} \\
\hline & $\left\|e_{h}^{N}\right\|_{\infty}$ & Error - rate & $\left\|e_{h}^{N}\right\|_{\infty}$ & Error - rate & $\left\|e_{h}^{N}\right\|_{\infty}$ & Error - rate \\
\hline $2^{-4}$ & 0.0299 & - & 0.0351 & - & 0.0565 & - \\
\hline $2^{-5}$ & 0.0155 & 1.9290 & 0.0185 & 1.8973 & 0.0308 & 1.8344 \\
\hline $2^{-6}$ & 0.0079 & 1.9620 & 0.0096 & 1.9271 & 0.0164 & 1.8781 \\
\hline $2^{-7}$ & 0.0040 & 1.9750 & 0.0049 & 1.9592 & 0.0087 & 1.8851 \\
\hline $2^{-8}$ & 0.0020 & 2.0000 & 0.0025 & 1.9600 & 0.0047 & 1.8510 \\
\hline
\end{tabular}

$$
B_{K}=\left[\begin{array}{ccccc}
0 & t_{1-K} & \cdots & t_{-2} & t_{-1} \\
t_{K-1} & 0 & t_{1-K} & \ddots & t_{-2} \\
\vdots & t_{K-1} & 0 & \ddots & \cdots \\
t_{2} & \ddots & \ddots & \ddots & t_{1-K} \\
t_{1} & t_{2} & \cdots & t_{K-1} & 0
\end{array}\right]
$$

It is known that a circulant matrix $C_{2 K}$ can be decomposed as follows $[19,20]$ :

$$
C_{2 K}=F_{2 K}^{-1} \operatorname{diag}\left(F_{(2 K)} c_{2 K}\right) F_{2 K},
$$

where $c_{2 K}$ is the first column vector of $C_{2 K} \cdot F_{2 K}$ is the $2 K \times$ $2 K$ discrete Fourier transform matrix in which $(j, k)$-entry $\left(F_{2 K}\right)_{j, k}$ of the matrix $F_{2 K}$ is given by

$$
\begin{aligned}
& \left(F_{2 K}\right)_{j, k}=\frac{1}{2 K} \exp \left(\frac{2 \pi i j k}{2 K}\right), \\
& \quad i=\sqrt{-1}, 0 \leq j, k \leq 2 K-2 .
\end{aligned}
$$

For any vector $\mu_{K} \in R^{K}$, we extend the vector by 0 to a $2 K$-dimensional vector $\mu_{2 K}=\left(\begin{array}{c}\mu_{K} \\ 0\end{array}\right)$. It is well known that the matrix-vector multiplication $F_{2 K} \mu_{2 K}$ can be carried out in $O(2 K \log 2 K)=O(K \log K)$ operations via the fast Fourier transform. Equation (33) shows that $F_{2 K} \mu_{2 K}$ can be evaluated in $O(K \log K)$ operations. We observe

$$
C_{2 K}\left(\begin{array}{c}
\mu_{K} \\
0
\end{array}\right)=F_{2 K}^{-1} \operatorname{diag}\left(F_{2 K} c_{2 K}\right) F_{2 K}\left(\begin{array}{c}
\mu_{K} \\
0
\end{array}\right) \text {. }
$$

At the same time,

$$
C_{2 K}\left(\begin{array}{c}
\mu_{K} \\
0
\end{array}\right)=\left(\begin{array}{cc}
T_{K} & B_{K} \\
B_{K} & T_{K}
\end{array}\right)\left(\begin{array}{c}
\mu_{K} \\
0
\end{array}\right)=\left(\begin{array}{c}
T_{K} \mu_{K} \\
B_{K} \mu_{K}
\end{array}\right) .
$$

Thus, the upper half of the vector multiplication $F_{2 K} \mu_{2 K}$ gives the matrix-vector multiplication $T_{K} \mu_{K}$. So we can evaluate $T_{K} \mu_{K}$ in $O(K \log K)$ operations. Then, by (28) we evaluate $A_{K-1, K-1} \mu_{K-1}$ in $O(K \log K)$ operations. Then, from (27), for any vector $\mu_{K} \in R^{K}$, the matrix-vector multiplication $T_{K} \mu_{K}$ is evaluated in $O(K \log K)$ operations without any lossy compression.

Remark 7. If the system matrix $A$ from (10) is a dense and full coefficient matrix, the Gaussian elimination method

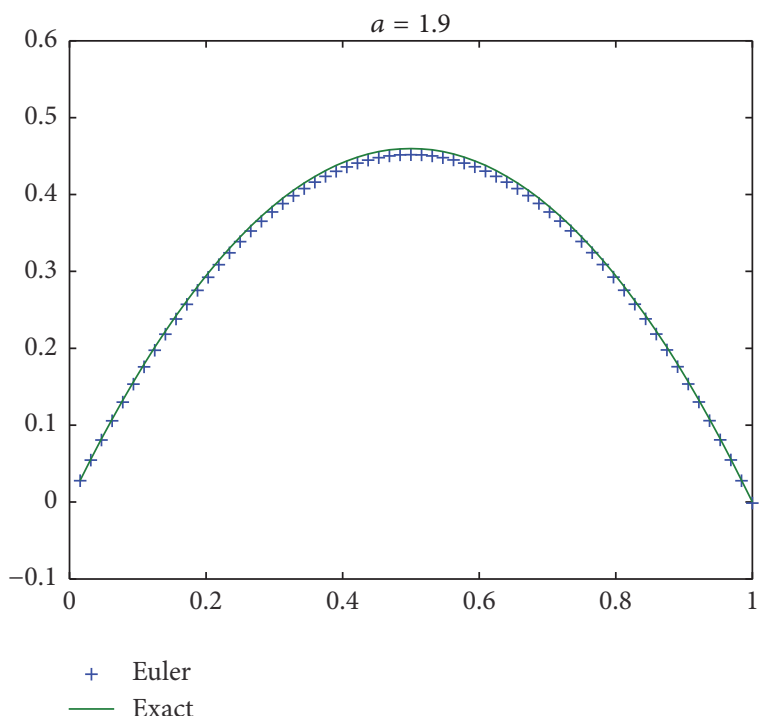

FIGURE 1: The behavior solution at $T=1$ with $\Delta t=h=2^{-6}, \alpha=1.9$.

requires storage of $O\left(K^{2}\right)$ and computational cost of $O\left(K^{3}\right)$. In this paper, the system matrix $A$ is supertriangular; the traditional Gaussian elimination method requires storage of $O\left(K^{2}\right)$ and computational cost of $O\left(K^{2}\right)$. We present a fast iterative method for the implicit finite difference scheme, which only requires storage of $O(K)$ and computational cost of $O(K \log K)$.

\section{A Numerical Example}

The following fractional differential equation was considered:

$$
\frac{\partial p(r, t)}{\partial t}=-v(r) \frac{\partial p(r, t)}{\partial r}+d(r) \frac{\partial^{\alpha} p(r, t)}{\partial r^{\alpha}}+f(r, t)
$$

On a finite domain $[0,1]$. The flow velocity is $v(r)=$ $\alpha /(4-2 \alpha)$. Diffusion coefficient is $d(r)=\Gamma(2-\alpha) \cdot r^{\alpha-1}$. The source term is $f(r, t)=5 e^{-t}\left(r^{2}+(3 \alpha-4) /(4-2 \alpha)\right)$. The initial function is $u(r)=5 r(1-r)$. And the boundary conditions are $w(t)=5 e^{-t}(1 /(2-\alpha)-2 /(3-\alpha)(2-\alpha))$.

Note that the exact solution to this problem is $p(r, t)=$ $5 e^{-t} r(1-r)$. In the numerical experiments, we consider three different $\alpha$ in the case, respectively. Table 1 shows $\left\|e_{h}^{N}\right\|_{\infty}$ the maximum error of the numerical solution of at the time $T=1$ and $\beta=1.0$. The stability and its order $O(\Delta t+h)$ of convergence are proved.

Figures 1-3 show the numerical solutions obtained by applying the Euler method (10) discussed above, with $\alpha=1.9$, 


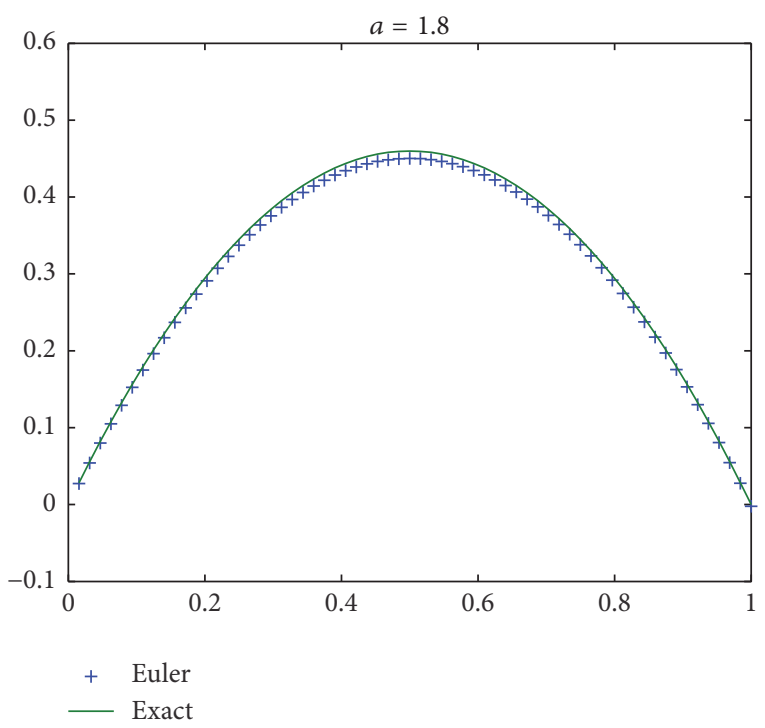

FIgURE 2: The behavior solution at $T=1$ with $\Delta t=h=2^{-6}, \alpha=1.8$.

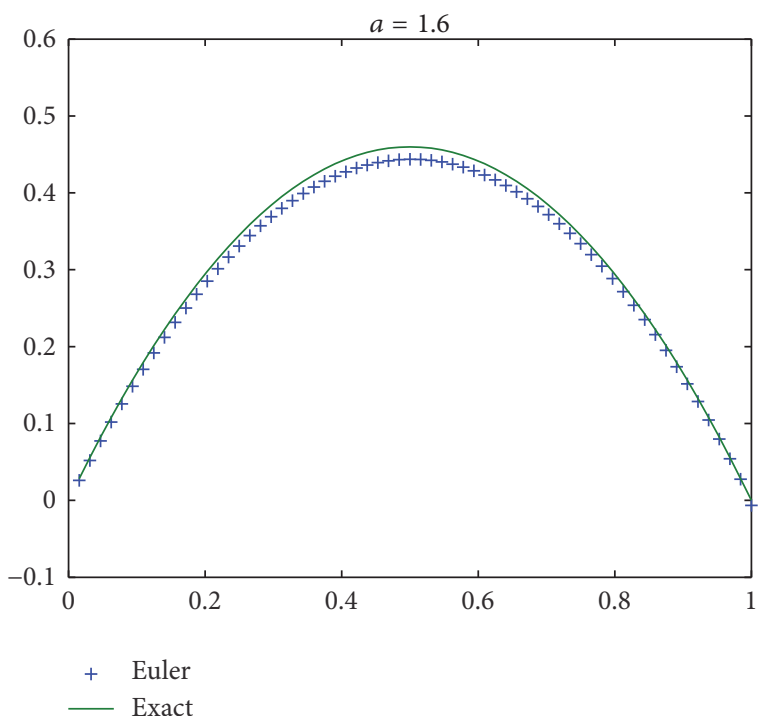

FIGURE 3: The behavior solution at $T=1$ with $\Delta t=h=2^{-6}, \alpha=1.6$.

$\alpha=1.8$, and $\alpha=1.6$ respectively, with $T=1, \beta=1.0$ and $\Delta t=h=2^{-6}$. The numerical solution compares well with the exact analytic solution to the fractional partial differential equation in these test cases.

\section{Conflicts of Interest}

The authors declare that there are no conflicts of interest regarding the publication of this paper.

\section{Acknowledgments}

This work was supported by the National Science Foundation of China under Grants 61375063, 61271355, 11301549, and 11271378 .

\section{References}

[1] K. S. Miller and B. Ross, An Introduction to the Fractional Calculus and Fractional Differential Equations, John Wiley \& Sons, New York, NY, USA, 1993.

[2] S. G. Samko, A. A. Kilbas, and O. I. Marichev, Frcational Integrals and Derivatives: Theory and Applications, Gordon Breach, London, Uk, 1993.

[3] I. M. Sokolov, J. Klafter, and A. Blumen, "Fractional kinetics," Physics Today, vol. 55, no. 11, pp. 48-54, 2002.

[4] R. L. Magin, Fractional Calculus in Bioengineering, Begell House Publishers, Danbury, Conn, USA, 2006.

[5] M. Raberto, E. Scalas, and F. Mainardi, "Waiting-times and returns in high-frequency financial data: an empirical study," Physica A: Statistical Mechanics and Its Applications, vol. 314, no. 1-4, pp. 749-755, 2002.

[6] X. Liu and Z. Liu, "Existence results for fractional semilinear differential inclusions in Banach spaces," Journal of Applied Mathematics and Computing, vol. 42, no. 1-2, pp. 171-182, 2013.

[7] X. Liu and Z. Liu, "On the 'bang-bang' principle for a class of fractional semilinear evolution inclusions," Proceedings of the Royal Society of Edinburgh Section A: Mathematics, vol. 144, no. 2, pp. 333-349, 2014.

[8] X. Liu, Z. Liu, and X. Fu, "Relaxation in nonconvex optimal control problems described by fractional differential equations," Journal of Mathematical Analysis and Applications, vol. 409, no. 1, pp. 446-458, 2014.

[9] X. Yin, L. Li, and S. Fang, "Second-order accurate numerical approximations for the fractional percolation equations," The Journal of Nonlinear Sciences and Applications, vol. 10, no. 08, pp. 4122-4136, 2017.

[10] H. Chen, S. Gan, and D. Xu, "A fractional trapezoidal rule type difference scheme for fractional order integro-differential equation," Journal of Fractional Calculus and Applications, vol. 7, no. 1, pp. 133-146, 2016.

[11] H. Chen, S. Gan, D. Xu, and Q. Liu, "A second-order BDF compact difference scheme for fractional-order Volterra equation," International Journal of Computer Mathematics, vol. 93, no. 7, pp. 1140-1154, 2016.

[12] I. Podlubny, Fractional Differential Equations, vol. 198 of Mathematics in Science and Engineering, Academic Press, San Diego, Calif, USA, 1999.

[13] D. A. Benson, S. W. Wheatcraft, and M. M. Meerschaert, "Application of a fractional advection-dispersion equation," Water Resources Research, vol. 36, no. 6, pp. 1403-1412, 2000.

[14] M. M. Meerschaert and C. Tadjeran, "Finite difference approximations for fractional advection-dispersion flow equations," Journal of Computational and Applied Mathematics, vol. 172, no. 1, pp. 65-77, 2004.

[15] J. Jia and H. Wang, "Fast finite difference methods for spacefractional diffusion equations with fractional derivative boundary conditions," Journal of Computational Physics, vol. 293, pp. 359-369, 2015.

[16] B. Guo, Q. Xu, and Z. Yin, "Implicit finite difference method for fractional percolation equation with Dirichlet and fractional boundary conditions," Applied Mathematics and Mechanics. English Edition, vol. 37, no. 3, pp. 403-416, 2016.

[17] L. Feng, P. Zhuang, F. Liu, I. Turner, and Q. Yang, "Secondorder approximation for the space fractional diffusion equation with variable cofficient," Progress in Fractional Differentiation and Applications, vol. 1, pp. 23-35, 2015. 
[18] A. Böttcher and B. Silbermann, Introduction to Large Truncated Toeplitz Matrices, Springer, New York, NY, USA, 1999.

[19] R. M. Gray, “Toeplitz and circulant matrices: a review," Foundations and Trends in Communications and Information Theory, vol. 2, no. 3, pp. 155-239, 2006.

[20] P. J. Davis, Circulant Matrices, John Wiley \& Sons, New York, NY, USA, 1979. 


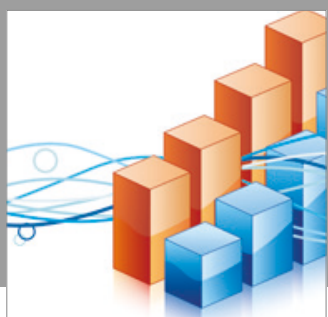

Advances in

Operations Research

vatersals

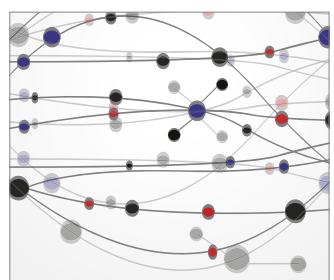

\section{The Scientific} World Journal
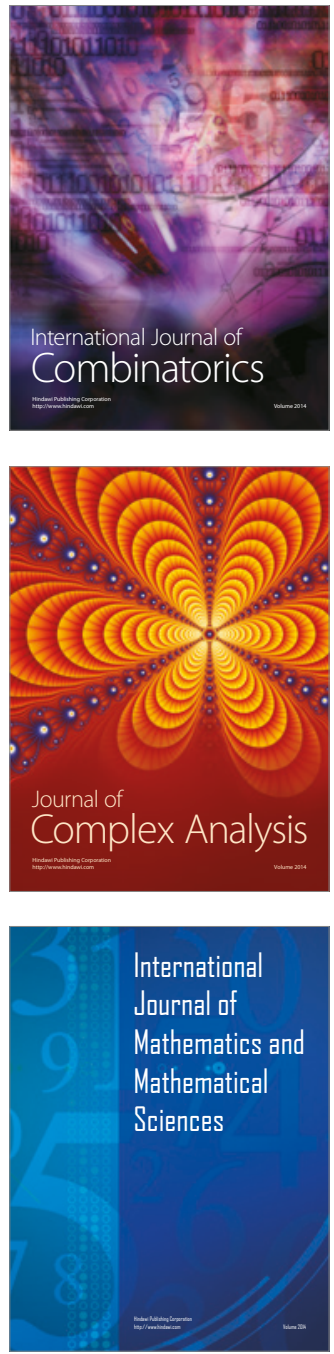
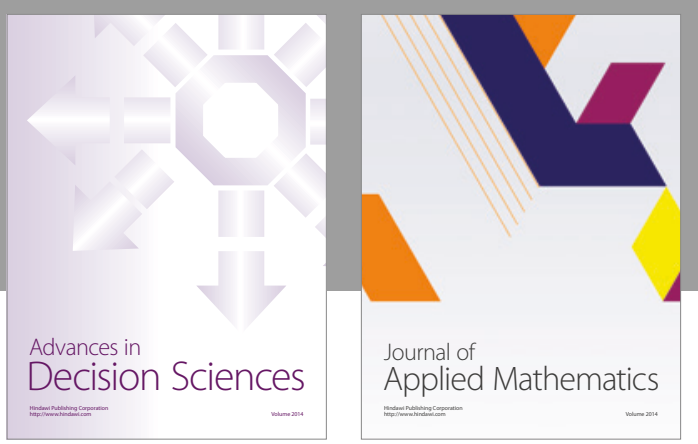

Algebra

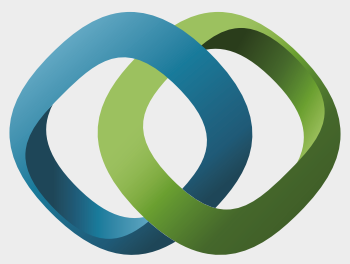

\section{Hindawi}

Submit your manuscripts at

https://www.hindawi.com
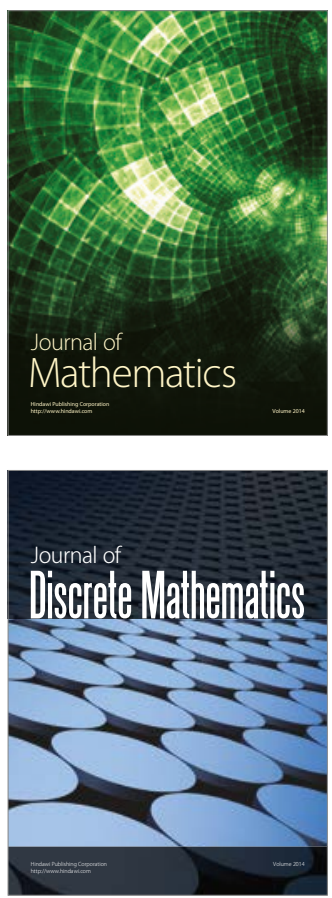

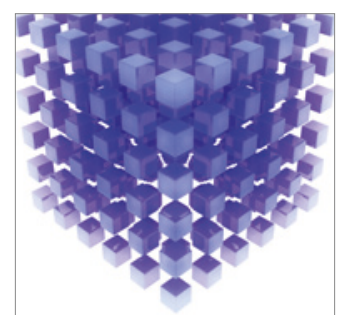

Mathematical Problems in Engineering
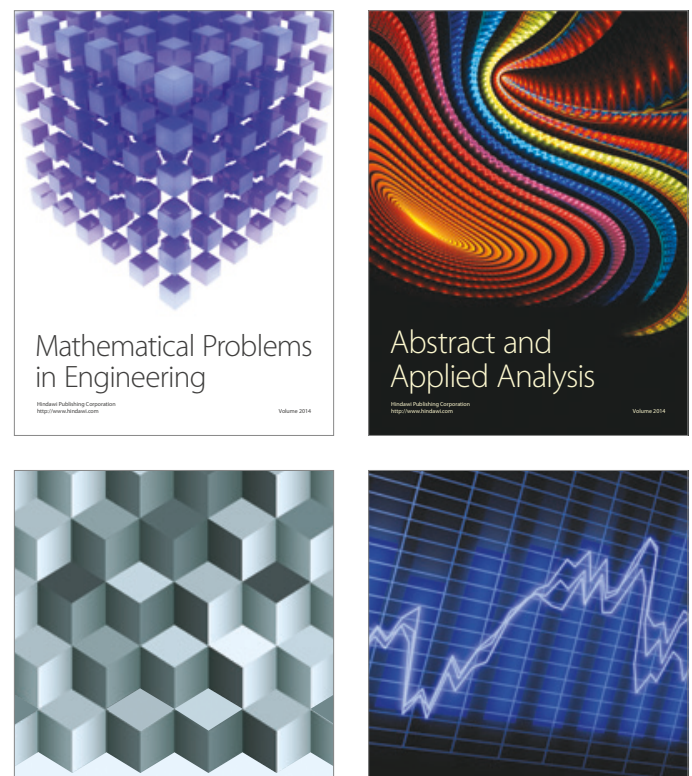

Journal of

Function Spaces

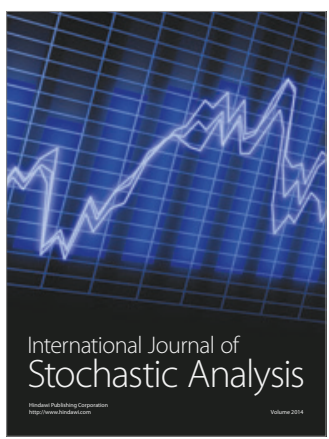

Probability and Statistics
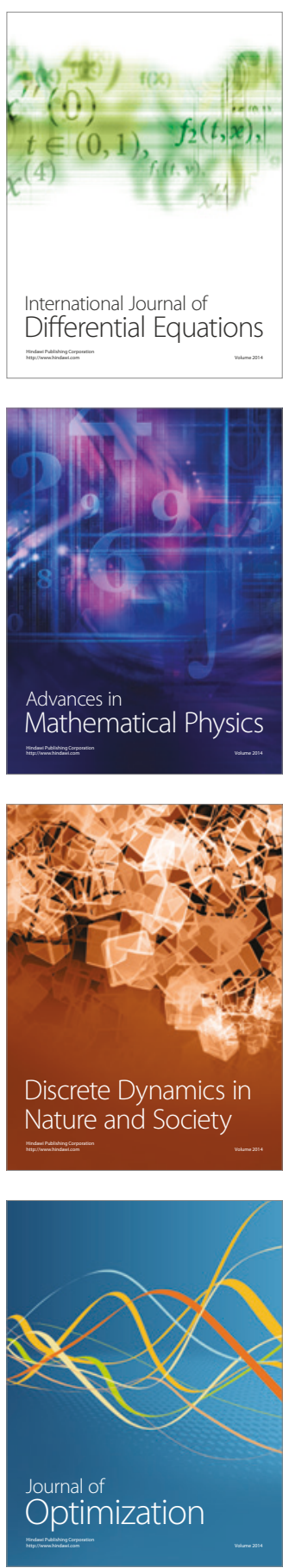